\title{
Hypertensive retinopathy and incident coronary heart disease in high risk men
}

\author{
B B Duncan, T Y Wong, H A Tyroler, C E Davis, F D Fuchs
}

See end of article for authors' affiliations

Correspondence to

Tien Yin Wong,

Department of

Ophthalmology, National

University of Singapore,

10 Kent Ridge Crescent,

Singapore 119260;

ophwty@nus.edu.sg

Accepted for publication

1 May 2002
Br J Ophthalmol 2002;86:1002-1006

Background/aim: Although routine ophthalmoscopy is recommended in the evaluation of people with hypertension, the prognostic significance of retinopathy is unknown. The purpose of this study is to determine if hypertensive retinopathy predicts coronary heart disease (CHD).

Methods: A prospective cohort study involving 560 hypertensive, hyperlipidaemic, middle aged men enrolled in the Lipid Research Clinic's Coronary Primary Prevention Trial. Signs of hypertensive retinopathy (generalised and focal arteriolar narrowing, arteriovenous nicking, widened arteriolar light reflex, retinal haemorrhage and exudates, microaneurysms, and disc swelling) were evaluated by direct funduscopy during a baseline examination by study physicians. Incident CHD events were ascertained from hospital records, necropsy reports, and death certificates, and reviewed by a masked panel of cardiologists.

Results: There were 51 definite CHD events (definite CHD deaths or myocardial infarctions) during a median follow up of 7.8 years. After adjusting for age, blood pressure, electrocardiographic manifestations of left ventricular hypertrophy, cholesterol levels and treatment, glucose and creatinine levels, and smoking status in proportional hazards analysis, the presence of hypertensive retinopathy predicted a doubling of the risk of definite CHD events (relative risk $2.1 ; 95 \%$ confidence interval (Cl) 1.0 to 4.2 ). The presence of either generalised or focal arteriolar narrowing predicted almost a tripling of the risk (relative risk $2.9 ; 95 \% \mathrm{Cl} 1.3$ to 6.2). Associations were similar for stage 1 hypertension (systolic and diastolic blood pressures of 140-159 and 90-99 mm Hg, respectively) and for other $\mathrm{CHD}$ end points.

Conclusion: Hypertensive retinopathy predicts CHD in high risk men, independent of blood pressure and CHD risk factors. The data support the concept that retinal microvascular changes are markers of blood pressure damage and may be useful in risk stratification and in the tailoring of hypertension treatment decisions.
$\mathrm{T}$ he Joint National Committee on the Prevention, Detection, Evaluation, and Treatment of High Blood Pressure in the United States and the British Society of Hypertension recommend routine ophthalmoscopic examination to detect signs of retinopathy in people with hypertension for the purposes of risk stratification and treatment decisions. ${ }^{12}$ However, little is known about the prognostic significance of retinopathy in hypertensive individuals. ${ }^{3}$ A number of studies have indicated that hypertensive individuals with retinopathy have higher mortality rates, and possibly higher morbidity rates from cardiovascular diseases. ${ }^{4-10}$ However, these studies have important limitations. Firstly, the majority were conducted before 1970, and it is uncertain if the data still apply to contemporary populations. For example, the more severe retinal changes described in these studies (for example, soft exudates/cotton wool spots and optic disc swelling) have been shown to be relatively uncommon in populations with better blood pressure control. ${ }^{11}$ Secondly, although retinopathy was shown to be predictive of overall mortality in these studies, specific cardiovascular outcomes (such as incident CHD, or CHD mortality) have not been evaluated. Further, the effect of potential confounders of any retinopathy-cardiovascular disease association (such as blood pressure level, clinically detected left ventricular hypertrophy or renal damage, smoking and lipid levels) is not known. Thus, clinical data on whether retinopathy provides useful predictive information for the purpose of CHD risk stratification beyond that already obtained by measuring blood pressure and lipid levels are not currently available. Partly because of these limitations, the value of retinal evaluation in people with hypertension has been questioned. ${ }^{12-15}$
The purpose of this study was to investigate whether hypertensive retinopathy, as diagnosed by physicians using direct ophthalmoscopy, predicts the long term incidence of CHD, independent of blood pressure and other cardiovascular risk factors. Hypertensive men participating in the Lipid Research Clinics Coronary Primary Prevention Trial (LRC-CPPT) provided the basis for this investigation.

\section{METHODS}

\section{Study population}

The LRC-CPPT cohort and procedures have been described elsewhere. ${ }^{16-19}$ Briefly, between 1973 and 1976, a total of 3806 men aged 35-59 years with type II-a hyperlipidaemia (total cholesterol $>6.85 \mathrm{mmo} / \mathrm{l}$ or $265 \mathrm{mg} / \mathrm{dl}$, low density lipoprotein cholesterol (LDL-cholesterol) $>4.91 \mathrm{mmol} / \mathrm{l}$ or $190 \mathrm{mg} / \mathrm{dl}$, and triglycerides $<3.39 \mathrm{mmol} / \mathrm{l}$ or $300 \mathrm{mg} / \mathrm{dl}$ ), and without clinically apparent CHD were randomised into two treatment groups, one receiving cholestyramine resin and the other an inert placebo. Though recruitment involved both clinical and community based approaches, most participants were recruited through community based strategies. Additional exclusion criteria included blood pressure $=180 / 120 \mathrm{~mm} \mathrm{Hg}$ at one of the four prerandomisation visits, or $=160 / 105 \mathrm{~mm} \mathrm{Hg}$ at more than one of the last three of these prerandomisation visits; the use of antihypertensive medication, a history of stroke or transient ischaemic attack; significant peripheral vascular disease (previous surgery or symptoms limiting treadmill examination); aortic aneurysm; diabetes mellitus (fasting glucose $>130 \mathrm{mg} / \mathrm{dl}$ or receiving insulin or oral hypoglycaemics); thyroid disease; nephrotic syndrome; the 
Table 1 Baseline characteristics comparing men with definite CHD $(n=51)$ versus those without definite CHD ( $n=509)$, participating in the Lipid Research Clinics Coronary Primary Prevention Trial

\begin{tabular}{|c|c|c|c|}
\hline \multirow[b]{2}{*}{ Characteristic at baseline } & Definite CHD & No definite CHD & \multirow[b]{2}{*}{$\mathrm{p}$ Value } \\
\hline & Mean (SE)* & Mean (SE)* & \\
\hline Age (years) & $51.3(0.9)$ & $48.9 \quad(0.3)$ & 0.01 \\
\hline Systolic blood pressure $(\mathrm{mm} \mathrm{Hg})$ & $139.3 \quad(1.3)$ & $137.7 \quad(0.4)$ & 0.24 \\
\hline Diastolic blood pressure $(\mathrm{mm} \mathrm{Hg})$ & $92.2(0.8)$ & $92.7 \quad(0.3)$ & 0.60 \\
\hline Total cholesterol (mg/dl) & $292.9 \quad(4.6)$ & $295.1 \quad(1.4)$ & 0.65 \\
\hline LDL-cholesterol (mg/dl) & $214.8 \quad(4.4)$ & $215.7 \quad(1.4)$ & 0.85 \\
\hline HDL-cholesterol (mg/dl) & $42.1 \quad(1.6)$ & $46.0 \quad(0.5)$ & 0.02 \\
\hline Creatinine $(\mathrm{mg} / \mathrm{dl})$ & $1.03(0.02)$ & $1.03(0.01)$ & 0.83 \\
\hline Fasting glucose (mg/dl) & $98.9(1.4)$ & $100.5(0.4)$ & 0.26 \\
\hline Average cigarettes smoked $\ddagger$ & $29.3 \quad(2.9)$ & $24.2 \quad(1.2)$ & 0.10 \\
\hline
\end{tabular}

*SE $=$ standard error

$\dagger p$ Values are based on age adjusted comparison of mean values between men with versus without definite $\mathrm{CHD}$ (except for age).

†Excluding never smokers.

presence of a debilitating disease; serum creatinine $=176.8$ $\mu \mathrm{mol} / \mathrm{l}$ or $2 \mathrm{mg} / \mathrm{dl}$ or a urine protein concentration of $2+$; and left ventricular hypertrophy, as defined electrocardiographically by an Estes score of $5 .^{20}$

This analysis is confined to the 588 individuals characterised as having hypertension on the basis of a mean systolic and diastolic blood pressure at visits 1 and 2 of 140 or $90 \mathrm{~mm}$ $\mathrm{Hg}$, respectively. Of these, $28(4.8 \%)$ were excluded owing to either a missing ophthalmoscopic examination or ungradeable retina, leaving 560 for further analyses.

\section{Procedures}

The procedures and criteria for CHD case definitions have been published elsewhere. ${ }^{16}{ }^{18}$ The median in-trial period was 7.8 years with bimonthly follow up and yearly physical examinations (up to 1983). Medical records, electrocardiograms, death certificates, and necropsy records were reviewed for all participants. CHD was ascertained by a blinded panel of cardiologists according to a standardised protocol described previously. ${ }^{16}{ }^{18}$

After reviewing the patient's blood pressure and obtaining a history oriented towards cardiovascular disease, study physicians performed direct ophthalmoscopy without mydriasis at the beginning of a general physical examination. The following individual lesions were documented as present versus absent: generalised and focal arteriolar narrowing, arteriovenous nicking, widened arteriolar light reflex, retinal haemorrhage and exudates, microaneurysms and disc swelling. Hypertensive retinopathy was defined to include the presence of any one of the above lesions.

\section{Definitions and statistical analysis}

The primary end point in this analysis is a definite CHD event, defined as either a definite CHD death or a definite myocardial infarction. Additionally, the LRC classified suspected CHD events and any coronary event. ${ }^{16}{ }^{18}$ Individual retinal lesions were analysed separately, and an aggregate variable, any retinopathy, was defined as the presence of any of the above mentioned specific retinal lesions. A separate aggregate retinopathy variable was created excluding a widened light reflex, because this was felt to be the most subjective of all the retinal signs. Cox proportional hazards regression models ${ }^{21}$ were constructed to estimate the relative risk (RR) of $\mathrm{CHD}$, in the presence or absence of retinopathy, adjusting for (1) age, (2) age and hypertension indicators (systolic blood pressure, diastolic blood pressure, creatinine levels, and left ventricular hypertrophy score, based on electrocardiographic findings ${ }^{21}$ ), and (3) age, hypertension indicators, and other CHD risk factors used in risk stratification of hypertensive individuals (total cholesterol, LDL-cholesterol, high density lipoprotein (HDL)-cholesterol, current or exsmoking status, and fasting glucose levels at baseline) as well as cholesterol treatment status. These were selected for inclusion in the models as they are risk factors that might be already considered in the management decision to treat a hypertensive patient, based on current recommendations. ${ }^{1}$ Proportional hazards assumption was visually verified by plotting log of cumulative hazard function comparing presence or absence of any retinopathy over the 7.8 years of follow up. Next, analyses were repeated in men with stage 1 hypertension (defined as systolic blood pressure of 140-159 and diastolic blood pressure of $90-99 \mathrm{~mm} \mathrm{Hg}^{1}$ ) and for other CHD end points (definite CHD deaths, definite myocardial infarction, suspected and definite CHD, and any coronary event). Finally, the principal retinal variables were entered simultaneously in a model to evaluate whether the predictive ability of each retinal lesion was independent of that of the others.

\section{RESULTS}

Of the 560 men included in this study, 498 (88.9\%) had stage 1 hypertension (140-159/90-99 $\mathrm{mm} \mathrm{Hg}$ ) and 62 (11.1\%) stage 2 (160-169/100-109 mm Hg) disease. At baseline, 65 (11.6\%) participants had signs of retinopathy. Of the specific lesions detected, $32(5.7 \%)$ had generalised arteriolar narrowing, eight $(1.4 \%)$ focal arteriolar narrowing, 18 (3.2\%) arteriovenous nicking, and $20(3.6 \%)$ a widened arteriolar light reflex. One individual was found to have microaneurysms, without retinal haemorrhages, retinal exudates, or disc swelling. With respect to other CHD risk factors, 261 (47\%) were current and 154 (28\%) were ex-smokers; and 18 (3.2\%) had an Estes score of 4, suggesting possible left ventricular hypertrophy.

During the 7.8 median years of follow up, 51 men $(9.1 \%)$ had a definite CHD event. Of these, $43(7.7 \%)$ had a definite myocardial infarction and $13(2.3 \%)$ a definite CHD death (events not mutually exclusive, five men had both a definite myocardial infarction followed by a definite CHD death). In addition, 18 men (3.2\%) had a suspected CHD event. Summing these with other coronary end points, a total of 171 men $(30.5 \%)$ had a coronary event. In general, baseline characteristics were similar in participants with $(n=51)$ versus without $(n=509)$ definite CHD events (Table 1$)$, except that men with definite CHD events were older, and had lower baseline HDL-cholesterol levels).

The age adjusted RRs of CHD for retinal findings are shown in Table 2 . The presence of any retinopathy predicted a twofold higher risk for definite CHD (RR 2.1; 95\% CI 1.1 to 4.1 ), for its subcategory definite myocardial infarction (RR 2.0; 95\% CI 1.0 
Table 2 Age adjusted relative risk (RR) of coronary heart disease (CHD) death or myocardial infarction (MI), by the presence of retinal abnormalities, among hypertensive participants of the LRC-CPPT

\begin{tabular}{|c|c|c|c|c|c|c|c|}
\hline \multirow[b]{2}{*}{ Retinal abnormalities } & \multirow[b]{2}{*}{$\mathrm{N}$} & \multirow[b]{2}{*}{$\%$} & \multicolumn{3}{|l|}{ Definite CHD } & \multirow[b]{2}{*}{$\begin{array}{l}\text { Definite or } \\
\text { suspected CHD }\end{array}$} & \multirow[b]{2}{*}{$\begin{array}{l}\text { Any coronary } \\
\text { event }\end{array}$} \\
\hline & & & Death or MI & MI only & $\begin{array}{l}\text { Death or Ml in } \\
\text { men with stage } \\
1 \text { hypertension* }\end{array}$ & & \\
\hline Any retinopathy & 65 & 11.6 & 2.1 (1.1 to 4.1$)$ & 2.0 (1.0 to 4.2$)$ & $2.2(1.0$ to 4.5$)$ & 1.8 (1.0 to 3.2 ) & $1.3(0.8$ to 2.0$)$ \\
\hline Any retinopathy, except widened light reflex & 51 & 9.1 & 2.1 (1.0 to 4.3$)$ & 1.9 (0.8 to 4.2$)$ & $2.2(1.0$ to 4.8$)$ & 1.8 (1.0 to 3.5$)$ & $1.3(0.8$ to 2.0$)$ \\
\hline $\begin{array}{l}\text { Arteriolar narrowing } \\
\text { Generalised or focal }\end{array}$ & 38 & 6.8 & 3.1 (1.5 to 6.3$)$ & 2.7 (1.2 to 6.2 ) & $3.1(1.4$ to 7.1$)$ & 2.7 (1.4 to 5.2$)$ & $1.6(1.0$ to 2.6$)$ \\
\hline Generalised & 32 & 5.7 & 2.6 (1.2 to 5.8$)$ & $2.1(0.8$ to 5.4$)$ & 2.5 (1.0 to 6.3 ) & 2.5 (1.2 to 5.0 ) & $1.6(1.0$ to 2.8$)$ \\
\hline Focal & 8 & 1.4 & $2.9(0.7$ to 12.1$)$ & $3.4(0.8$ to 14.2$)$ & $4.0(1.0$ to 16.8$)$ & $2.1(0.5$ to 8.5$)$ & $0.7(0.2$ to 3.0$)$ \\
\hline Arteriovenous nicking & 18 & 3.2 & $1.8(0.6$ to 5.7$)$ & $1.4(0.3$ to 5.6$)$ & 2.9 (0.9 to 9.2$)$ & $1.7(0.6$ to 4.8$)$ & $1.0(0.4$ to 2.2$)$ \\
\hline Widened light reflex & 20 & 3.6 & $1.7(0.5$ to 5.4$)$ & $2.0(0.6$ to 6.4$)$ & $1.7(0.5$ to 5.6$)$ & 1.2 (0.4 to 3.8$)$ & $1.1(0.5$ to 2.4$)$ \\
\hline
\end{tabular}

Figures are age adjusted RRs. Figures in parentheses denote $95 \%$ confidence intervals.

*Defined by systolic and diastolic blood pressure levels of 140-159 and 90-99 mm Hg, respectively.

Table 3 Multivariate relative risk (RR) of definite coronary heart disease (CHD) death or myocardial infarction (MI), by the presence of retinal abnormalities, adjusted for hypertension† and other CHD risk factors $\ddagger$, among hypertensive participants of the LRC-CPPT

\begin{tabular}{|c|c|c|c|c|c|c|}
\hline \multirow[b]{2}{*}{ Retinal abnormalities } & \multicolumn{2}{|l|}{ Death or MI } & \multicolumn{2}{|l|}{ MI only } & \multicolumn{2}{|c|}{$\begin{array}{l}\text { Death or } \mathrm{Ml} \text { in men with stage } 1 \\
\text { hypertension* }\end{array}$} \\
\hline & $\begin{array}{l}\text { RR, adjusted for } \\
\text { hypertensiont }\end{array}$ & $\begin{array}{l}\text { Multivariate } \\
\text { adjusted RR }\end{array}$ & $\begin{array}{l}\text { RR, adjusted for } \\
\text { hypertensiont }\end{array}$ & $\begin{array}{l}\text { Multivariate } \\
\text { adjusted RR }\end{array}$ & $\begin{array}{l}\text { RR, adjusted for } \\
\text { hypertensiont }\end{array}$ & $\begin{array}{l}\text { Multivariate } \\
\text { adjusted RR }\end{array}$ \\
\hline Any retinopathy & $2.2(1.1$ to 4.3$)$ & 2.1 (1.0 to 4.2$)$ & 2.0 (0.9 to 4.2$)$ & 1.9 (0.9 to 4.2$)$ & 2.1 (1.0 to 4.5$)$ & $2.0(0.9$ to 4.5$)$ \\
\hline $\begin{array}{l}\text { Any retinopathy except for widened light } \\
\text { reflex }\end{array}$ & $2.2(1.0$ to 4.6$)$ & $2.0(1.0$ to 4.3$)$ & $1.9(0.8$ to 4.3$)$ & 1.710 & $2.2(1.0$ to 5.0$)$ & $1.9(0.8$ to 4.5$)$ \\
\hline Arteriolar narrowing & & & & & & \\
\hline Generalised or focal & $3.3(1.5$ to 6.9$)$ & 2.9 (1.3 to 6.2$)$ & $2.8(1.2$ to 6.4$)$ & $2.4(1.0$ to 5.8$)$ & $3.1(1.3$ to 7.1$)$ & $2.6(1.1$ to 6.3$)$ \\
\hline Generalised only & $2.8(1.2$ to 6.5$)$ & $2.5(1.1$ to 5.9$)$ & $2.2(0.8$ to 5.8$)$ & $1.9(0.7$ to 5.2$)$ & 2.5 (0.9 to 6.5$)$ & $2.3(0.8$ to 6.1$)$ \\
\hline Arteriovenous nicking & $1.9(0.6$ to 6.2$)$ & $2.1(0.6$ to 6.9$)$ & $1.5(0.4$ to 6.3$)$ & $1.6(0.4$ to 6.9$)$ & $3.3(1.0$ to 10.9$)$ & $3.2(1.0$ to 10.7 \\
\hline Widened light reflex & $1.6(0.5$ to 5.3$)$ & $1.8(0.5$ to 6.1$)$ & $1.8(0.6$ to 6.0$)$ & $2.2(0.6$ to 7.6$)$ & $1.6(0.5$ to 5.2$)$ & $1.8(0.5$ to 6.1$)$ \\
\hline
\end{tabular}

*Defined by systolic and diastolic blood pressure levels of 140-159 and 90-99 mm Hg, respectively.

tAdjusted for age, systolic and diastolic blood pressure, creatinine levels, and left ventricular hypertrophy score at baseline.

$\ddagger$ Adjusted for above factors plus total cholesterol, LDL-cholesterol, HDL-cholesterol, cholesterol treatment status, current or ex-smoking status, and fasting glucose levels at baseline. Figures in parentheses denote $95 \%$ confidence intervals.

to 4.2) and also for definite CHD among men with stage 1 hypertension only (RR $2.2 ; 95 \%$ CI 1.0 to 4.5 ). These findings did not vary significantly after excluding those with only a widened light reflex. The presence of arteriolar narrowing (either generalised or focal) predicted a nearly threefold higher risk of CHD. For specific retinal lesions and definite CHD, the RR ranged from 1.7 (95\% CI 0.5 to 5.4) for widened light reflex to 2.9 (95\% CI 0.7 to 12.1) for focal arteriolar narrowing, although only the association for generalised arteriolar narrowing was statistically significant (RR 2.6; 95\% CI, 1.2 to 5.8 ). When the data were analysed including suspected CHD events, the results were similar (Table 2 ). When any coronary event was the outcome of interest, the associations were weaker.
After adjusting for systolic and diastolic blood pressure, creatinine levels, and electrocardiographic manifestations of left ventricular hypertrophy, men with any retinopathy had 2.2 ( $95 \%$ CI, 1.1 to 4.3 ) times the risk of CHD than those without (Table 3). This association was not attenuated after further adjusting for other cardiovascular risk factors (RR of 2.1, 95\% CI, 1.0 to 4.2) and was similar to that found for the subcategory definite myocardial infarction alone. Likewise, the associations did not change after excluding those with only a widened light reflex, and were stronger for arteriolar narrowing (multivariate RRs of 2.9 for any narrowing and 2.5 for generalised narrowing, respectively). When the analysis was confined to men with stage 1 hypertension, the associations were of similar strength.

Table 4 Multivariate relative risk (RR) of definite coronary heart disease (CHD) death or myocardial infarction (MI) by individual retinal abnormalities when adjusted simultaneously for other retinal abnormalities, for hypertension $\dagger$ and other CHD risk factors $\ddagger$, among hypertensive participants of the LRC-CPPT

\begin{tabular}{|c|c|c|c|c|c|c|}
\hline \multirow[b]{2}{*}{ Retinal abnormalities } & \multicolumn{2}{|l|}{ Death or MI } & \multicolumn{2}{|l|}{ MI only } & \multicolumn{2}{|c|}{$\begin{array}{l}\text { Death or Ml in men with stage } 1 \\
\text { hypertension* }\end{array}$} \\
\hline & $\begin{array}{l}\text { RR, adjusted for } \\
\text { hypertensiont }\end{array}$ & $\begin{array}{l}\text { Multivariate } \\
\text { adjusted RR }\end{array}$ & $\begin{array}{l}\text { RR, adjusted for } \\
\text { hypertensiont }\end{array}$ & $\begin{array}{l}\text { Multivariate } \\
\text { adjusted RR }\end{array}$ & $\begin{array}{l}\text { RR, adjusted for } \\
\text { hypertension } \dagger\end{array}$ & $\begin{array}{l}\text { Multivariate } \\
\text { adjusted RR }\end{array}$ \\
\hline Generalised or focal arteriolar narrowing & $3.1(1.4$ to 6.8$)$ & 2.7 (1.2 to 5.9$)$ & $2.6(1.1$ to 6.3$)$ & $2.2(0.9$ to 5.5$)$ & $2.9(1.2$ to 7.0$)$ & 2.5 (1.0 to 6.2$)$ \\
\hline Arteriovenous nicking & $1.6(0.5$ to 5.3$)$ & 1.7 (0.5 to 5.8$)$ & $1.3(0.4$ to 4.5$)$ & $1.3(0.3$ to 5.8$)$ & $3.0(0.9$ to 10.5$)$ & 2.9 (0.8 to 10.2$)$ \\
\hline Widened light reflex & 1.1 (0.3 to 3.7 ) & $1.3(0.4$ to 4.7$)$ & 1.2 (0.3 to 5.3$)$ & $1.8(0.5$ to 6.5$)$ & 0.9 (0.3 to 3.3$)$ & $1.1(0.3$ to 4.4$)$ \\
\hline
\end{tabular}

*Defined by systolic and diastolic blood pressure levels of 140-159 and 90-99 mm Hg, respectively.

†Adjusted for age, systolic and diastolic blood pressure, creatinine levels, and left ventricular hypertrophy score at baseline.

$\ddagger$ Adjusted for above factors plus total cholesterol, LDL-cholesterol, HDL-cholesterol, cholesterol treatment status, current or ex-smoking status, and fasting glucose levels at baseline. Figures in parentheses denote $95 \%$ confidence intervals. 
Additional analyses were performed for other CHD end points, with similarly consistent results. For example, arteriolar narrowing predicted a higher risk of definite CHD deaths (age adjusted RR of 4.2; 95\% CI, 1.2 to 15.5 ) and any coronary events (multivariate RR $1.7 ; 95 \%$ CI 1.0 to 2.8 ). On the other hand, for non-CHD deaths $(n=13)$, no associations were found. Finally, in models with all retinal variables entered simultaneously (Table 4), generalised or focal arteriolar narrowing independently predicted a higher rate of definite CHD, other abnormalities predicting small, but not statistically significant, additional risk.

\section{DISCUSSION}

Hypertensive retinopathy consists of a spectrum of retinal vascular changes that are pathologically related to both transient and persistent microvascular damage from elevated blood pressure..$^{22}$ The relation between blood pressure and the presence and severity of theses changes are well established, and confirmed in several large population based studies. ${ }^{10}{ }^{11} 24-27$ The important issue of the prognostic significance of this retinopathy, however, has remained unclear. Does it merely reflect blood pressure levels (thus, providing no additional information for the management of hypertension over that obtained by measuring blood pressure); or does it offer important predictive information about cardiovascular health, beyond blood pressure measurement?

In this cohort of hypertensive, hyperlipidaemic, middle aged US men, the presence of retinopathy, as detected through a standard direct ophthalmoscopic examination performed by non-ophthalmologists, was associated with a twofold higher risk of CHD death or myocardial infarction, independent of systolic and diastolic blood pressure, left ventricular hypertrophy, creatinine levels, cholesterol levels and treatment, glucose levels, and smoking status. In fact, arteriolar narrowing, whether generalised or focal, was independently associated with a nearly three times higher risk of CHD. The higher risk persisted with diverse CHD end points, including definite CHD deaths, definite myocardial infarction, suspected and definite CHD, and any coronary event. Perhaps more importantly, the higher risk was also seen when analyses were restricted to those with only stage 1 hypertension, suggesting that retinal lesions are useful predictors even in people without severe hypertension. Although individual lesions had different strengths of association-strongest for arteriolar narrowing, weaker (and statistically non-significant) for arteriovenous nicking and a widened light reflex - the overall pattern and direction of all associations were consistent. Therefore, these data suggest that this retinopathy is an independent prognostic indicator of CHD risk in people with hypertension, and support the use of ophthalmoscopic examination for risk stratification, even for Stage 1 hypertension.

The current literature provides limited and inconsistent data on the association between retinopathy and either cardiovascular disease or cardiovascular mortality. ${ }^{3}$ Some studies have indicated that retinal changes may be associated with CHD even in people without hypertension. In a cross sectional study of 70 non-diabetic and non-hypertensive subjects undergoing coronary angiography, retinal lesions were associated with both the presence and severity of coronary artery disease..$^{28}$ In another cross sectional study based on data from the National Health Examination Survey, retinal changes were associated with a fourfold higher prevalence of CHD in white men aged 35-54 years and a sixfold higher prevalence in white women of similar age, after controlling for hypertension, diabetes, and cholesterol levels. ${ }^{29}$ Most recently, the Atherosclerosis Risk In Communities (ARIC) Study showed that retinal arteriolar narrowing was predictive of incident CHD, with stronger associations seen in women compared to men. ${ }^{30}$ It is not clear why the results of the ARIC study are different from the current study, although direct comparison of the two studies may be inappropriate, as the ARIC study was conducted among low risk men and women, and used retinal photographs for the assessment of retinal microvascular abnormalities.

In hypertensive people, the biological rationale for an association between hypertensive retinopathy and CHD is even stronger, as retinopathy results from arteriolar damage from elevated blood pressure, and it is reasonable to expect that microvascular damage in the retinal arterioles may be associated with concurrent microvascular damage in the coronary circulation as well. This was recognised as early as 1939 by Keith et al, when they showed a dose dependent increase in mortality with increasingly severe retinal changes. ${ }^{4}$ Although numerous studies have since documented possible associations between retinopathy and CHD in hypertensive individuals, these studies have significant limitations. ${ }^{4-10}$ These include possible inapplicability to current populations (for example, only one case in our cohort met the stage 3 hypertensive retinopathy definition proposed by Keith et al), inadequate evaluation of specific cardiovascular outcomes and lack of control for possible confounders. The current LRC study show that hypertensive/arteriosclerotic retinopathy is a predictor of CHD in high risk hypertensive men, independent of the increased risk associated with blood pressure level and other CHD risk factors.

A number of important limitations of this study should be mentioned. Firstly, it should be re-emphasised that the population was non-randomly selected, having been derived from a multicentre clinical trial, and consisted of hyperlipidaemic, middle age white men. Thus, caution should be taken when extending these findings to other segments of the population-those without hyperlipidaemia, older and younger age groups, women, other ethnic groups, etc. However, the fact that the association was minimally confounded by the presence of other risk factors (lipid levels, smoking, etc) and that cross sectional associations in the NHANES study ${ }^{29}$ were present in a representative sample of US males and, if anything, greater in a representative sample of US women, supports generalisation beyond those who participated in the trial. Secondly, medical advances in the recognition and treatment of hyperlipidaemia and hypertension, by altering the clinical course of these conditions, may have altered somewhat the predictive ability of retinal findings to current clinical practice. Thirdly, retinal assessment was performed using direct ophthalmoscopy on undilated eyes by non-ophthalmologists. Non-standardised direct ophthalmoscopic examination by physicians has been suggested to be less reliable, ${ }^{12-15}$ and to compare unfavourably with standardised photographic retinopathy grading systems, such as those used in the Beaver Dam Eye Study ${ }^{11}$ and the Atherosclerosis Risk in Communities Study. ${ }^{27}{ }^{30}{ }^{31}$ Finally, even though subjects with prevalent cardiovascular disease, repeated blood pressure measurement 160/105 mm Hg, or using antihypertension medication were excluded from the study, study physicians did review blood pressure and perform a medical history oriented toward cardiovascular disease immediately before the retinal examination. This knowledge could have influenced their determination of retinal findings. However, that our findings remained significant after adjustment for blood pressure level and the presence of other cardiovascular risk factors makes a resultant measurement bias less likely.

Significant strengths of this study should also be emphasised. Firstly, while standardised photographic grading in epidemiological research studies are inherently more valid and precise, they are currently not widely used for risk stratification in hypertensive individuals. ${ }^{3}$ In this regard, the lack of standardisation and quality control of the retinal examination should make our results clinically more relevant to standard medical practice. Secondly, cardiovascular outcomes in the LRC-CPPT study were rigorously validated and documented throughout the trial and follow up. ${ }^{16}$ Blood pressure and 
other covariates were also measured in standardised, quality controlled settings. Thirdly, as retinopathy is highly correlated with blood pressure, we adjusted all models not only for blood pressure, but creatinine levels and left ventricular hypertrophy, indicators of sustained elevated blood pressure. Thus, any residual confounding from hypertension is unlikely to be significant.

In conclusion, in hypertensive, hyperlipidaemic men, the presence of hypertensive retinopathy was associated with a twofold higher risk of CHD developing over a median of 7.8 years, independent of blood pressure levels and other coronary risk factors. Further studies of retinal microvascular abnormalities, perhaps with more objective methods such as retinal photography, ${ }^{30}{ }^{31}$ and in populations that are more representative of patients seen in current clinical practice, are indicated to define their prognostic significance more precisely. Nevertheless, these results are consistent with recent data that hypertensive retinal microvascular changes predict stroke, ${ }^{32}$ and support the current recommendation in the use of direct retinal examination for cardiovascular risk stratification.

\section{Authors' affiliations}

B B Duncan, Social Medicine Department, School of Medicine, Federal University of Rio Grande do Sul, Porto Alegre, RS Brazil

T Y Wong, Department of Ophthalmology, National University of Singapore, and Singapore National Eye Centre and Singapore Eye Research Institute, Singapore

H A Tyroler, Department of Epidemiology, School of Public Health, University of North Carolina, Chapel Hill, NC, USA

C E Davis, Department of Biostatistics, School of Public Health, University of North Carolina, Chapel Hill, NC, USA

F D Fuchs, Internal Medicine Department, School of Medicine, Federal University of Rio Grande do Sul, Porto Alegre, RS Brazil

\section{REFERENCES}

1 Joint National Committee on the Prevention, Detection, Evaluation, and Treatment of High Blood Pressure. Sixth Report. NIH publication no 98-4080, 1997

2 Ramsay LE, Williams B, Johnston GD, et al. British Hypertension Society guidelines for hypertension management 1999: summary. BM 1999;319:630-5

3 Wong TY, Klein R, Klein BEK, et al. Retinal microvascular abnormalities and their relations with hypertension, cardiovascular diseases and mortality. Surv Ophthalmol 2001;46:59-80.

4 Keith NM, Wagener HP, Barker NW. Some different types of essential hypertension: their course and prognosis. Am J Med Sci 1939:197:332-43.

5 Palmer RS, Loofbourow D, Doering CR. Prognosis in essential hypertension: eight-year follow-up study of 430 patients on conventiona medical treatment. N Engl Med J 1948;239:990-4.

6 Oglesby $\mathbf{P}$, Lepper $\mathrm{MH}$, Phelan $\mathrm{WH}$, et al. A longitudinal study of coronary heart disease. Circulation 1963;28:20-31.

7 Breslin DJ, Gifford RW Jr, Fairbairn JF II, et al. Prognostic importance of ophthalmoscopic findings in essential hypertension. JAMA 1966;195:335-8.

8 Breslin DJ, Gifford RW Jr, Fairbairn JF II. Essential hypertension: a twenty year follow-up study. Circulation 1966;33:87-97.
9 Ralph RA. Prediction of cardiovascular status from arteriovenous crossing phenomena. Ann Ophthalmol 1974;6:323-6.

10 Svardsudd K, Wedel H, Aurell E, et al. Hypertensive eye ground changes: prevalence, relation to blood pressure and prognostic importance. Acta Med Scand 1978;204:159-67.

11 Klein R, Klein BEK, Moss SE, et al. Hypertension and retinopathy, arteriolar narrowing, and arteriovenous nicking in a population. Arch Ophthalmol 1994;112:92-8.

12 Kagan A, Aurell E, Dobree J, et al. A note of signs in the fundus oculi and arteriolar hypertension: conventional assessment and significance. Bull World Health Organ 1966:55-60.

13 Walsh JB. Hypertensive retinopathy: description, classification, and prognosis. Ophthalmology 1982;89:1 127-31.

14 Dimmitt SB, West JN, Eames SM, et al. Usefulness of ophthalmoscopy in mild to moderate hypertension. Lancet 1989;1:1103-6.

15 Fuchs FD, Maestri M, Moreira FC, et al. A study of the usefulness of optic fundi examination of hypertensive patients in a clinical setting. $J$ Human Hypert 1995;9:547-51.

16 Lipid Research Clinics Program. The Coronary Primary Prevention Trial: design and implementation. J Chronic Dis 1979;32:609-31.

17 Lipid Research Clinics Program. Pre-entry characteristics of participants in the Lipid Research Clinics Primary Prevention Trial. J Chronic Dis 1983:36:467-79

18 Lipid Research Clinics Program. The Lipid Research Clinics Coronary Primary Prevention Trial results. I: Reduction in incidence or coronary heart disease. JAMA 1984;251:351-64.

19 Lipid Research Clinics Program. The Lipid Research Clinics Coronary Primary Prevention Trial results, II: the relationship of reduction in incidence of coronary heart disease to cholesterol lowering. JAMA 1984;251:365-74

20 Romhilt DW, Estes EH Jr. A point-score system for the ECG diagnosis of left ventricular hypertrophy. Am Heart J 1968;75:752-8.

21 Cox DR. Regression models and life tables. J R Stat Soc 1972;34:220.

22 Tso MOM, Jampol LM. Pathophysiology of hypertensive retinopathy. Ophthalmology 1982;89:1132-45

23 Tso MOM, Abrams GW, Jampol LM: Hypertensive retinopathy, choroidopathy, and optic neuropathy: a clinical and pathophysiological approach to classification. In: Singerman $L$, Jampol LM, eds. Retinal and choroidal manifestations of systemic disease. Baltimore: Williams and Wilkins, 1991:79-127

24 Klein R, Klein BEK, Moss SE. The relation of systemic hypertension to changes in the retinal vasculature. The Beaver Dam Eye Study. Trans Am Ophthalmol Soc 1997;95:329-50.

25 Sharp PS, Chaturvedi N, Wormald R, et al. Hypertensive retinopathy in Afro-Caribbeans and Europeans. Prevalence and risk factor relationships. Hypertension 1995; 25:1322-5.

26 Yu T, Mitchell P, Berry G, Li W, et al. Retinopathy in older persons without diabetes and its relationship to hypertension. Arch Ophthalmol $1998 ; 116: 83-9$

27 Sharrett AR, Hubbard LD, Cooper LS, et al. Retinal arteriolar diameters and elevated blood pressure. The Atherosclerosis Risk in Communities (ARIC) Study. Am J Epidemiol 1999;150:263-70.

28 Michelson EL, Morganroth J, Nichols CW, et al. Retinal arteriolar changes as an indicator of coronary artery disease. Arch Intern Med1979;139:1 139-41.

29 Gillum RF: Retinal arteriolar findings and coronary heart disease. Am Heart J 1991; 122:262-3.

30 Wong TY, Klein R, Sharrett AR, et al. Retinal arteriolar narrowing and risk of coronary heart disease in men and women: the Atherosclerosis Risk in the Communities Study. JAMA 2002;287: 1153-9.

31 Couper DJ, Klein R, Hubbard LD, et al Reliability of retinal photography in the assessment of retinal microvascular characteristics: the Atherosclerosis Risk in Communities Study. Am J Ophthalmol 2002; 133:78-88

32 Wong TY, Klein R, Couper DJ, et al. Retinal microvascular abnormalities and incident stroke: the Atherosclerosis Risk in Communities Study. Lancet 2001;358:1134-40. 